\title{
The Effect of Resonances on Diffusive Scattering
}

\author{
B. Elattari国, V. Kagalovsky, and H.A. Weidenmüller \\ Max-Planck-Institut für Kernphysik, 69029 Heidelberg, Germany
}

(November 20, 2018)

\begin{abstract}
The presence of resonances modifies the passage of light or of electrons through a disordered medium. We generalize random matrix theory to account for this effect. Using supersymmetry, we calculate analytically the mean density of states, and the effective Lagrangean of the generating functional for the two-point function. We show that the diffusion constant scales with the effective mean level spacing. The latter exhibits a resonance dip. These facts allow us to interpret experimental results on light scattering for different concentrations of resonant scatterers.
\end{abstract}

\section{INTRODUCTION AND MOTIVATION}

During the last decade, many phenomena related to the passage of waves through a disordered medium have been intensely studied. This holds both for electrons, i.e. for the amplitude waves of the Schrödinger equation [1], and for light, i.e. for classical electromagnetic waves [2]. As examples, we mention conductance fluctuations in mesoscopic probes, the analogous effect of speckle patterns in the transmission of light, and weak and strong localization effects including the enhanced backscattering of electrons or light from a disordered medium. For electrons, disorder is typically caused by impurities. For light, disorder is often produced artificially: A powder is immersed into some liquid. Random scattering occurs if the indices of refraction of powder and liquid are sufficiently different.

The present paper has been motivated by an experiment performed several years ago [3] on the scattering of light by a disordered medium. In this experiment, the powder used $\left(\mathrm{TiO}_{2}\right)$ consisted of grains with a size distribution centered around a diameter of $220 \mathrm{~nm}$. For such granules, a Mie resonance occurs close to the wavelength $\lambda \sim 630 \mathrm{~nm}$ of the laser light used in the experiment. This led to a resonance enhancement of the diffusive scattering. The effects of such enhancement were seen by comparing the diffusion constant $\mathcal{D}$ (determined from the intensity autocorrelation function versus frequency of the transmitted light), with the transport mean free path $l$ (determined either from weak localization, i.e. from enhanced backscattering, or from the dependence of the transmitted intensity on the length $L$ of the disordered sample). For the quasi one-dimensional geometry of the present experiment, one expects $\mathcal{D}$ and $l$ to obey the relation $\mathcal{D}=(1 / 3) v_{E} l$, with $v_{E}$ the energy transport velocity through the disordered medium. In the present case, the ratio $3 \mathcal{D} / l$ yielded a value $v_{E}=(5 \pm 1) 10^{7} \mathrm{~m} \mathrm{~s}^{-1}$ which is about an order of magnitude smaller than the phase velocity. This surprising result has been understood both for small concentration [3-9] and, more recently, also for strong concentration [10,11] of the scatterers. (In the latter case, the resonant structure in the frequency dependence of $\mathcal{D}$ disappears, and an overall decrease of $\mathcal{D}$ is observed [12]). Qualitatively speaking, the transport velocity is reduced because on its way through the medium, the energy is stored for some time in the Mie resonances.

The present paper goes beyond previous theoretical work in two ways. First, we present a unified theoretical framework in which both average and fluctuation properties of the transmitted and reflected waves can be calculated on the same footing. This is in contrast 
to approaches [3,10,11] which use the Bethe-Salpeter equation [3] or a mean-field approximation [10,11] for the calculation of the transport velocity $v_{E}$, and a diagrammatic impurity perturbation expansion for the intensity autocorrelation function. Second, we identify the energy dependence of the mean level density $\rho(E)$ as the culprit for the observed deviation from standard behavior. A simple argument which yields the same result as the analytical derivation and illuminates the physical content may be helpful at this point. In the case of electrons [1], the Thouless formula $g=E_{c} \rho(E)$ connects the average conductance $g$ with the Thouless energy $E_{c}=\hbar \mathcal{D} / L^{2}$ and the mean level density. The presence of numerous resonances with equal resonance energies $E_{1}$ leads to a local Breit-Wigner-shaped increase of $\rho(E)$ near $E_{1}$. Since $g$ is not affected by the presence of the resonances, the Thouless formula implies that $E_{c}$ and, hence, the diffusion constant $\mathcal{D}$ have a Breit-Wigner-like dip near $E_{1}$. In Refs. [13, 14] we have shown that within the framework of the supersymmetry formalism, the effective Lagrangeans for electrons and for classical light waves coincide. Hence, the argument just given applies likewise to scattering of light. With increasing concentration of scatterers, the dip in $\mathcal{D}$ widens and becomes less deep. Eventually, this results in an overall decrease of $\mathcal{D}$ over a wide frequency interval. We emphasize that in the context of the supersymmetry approach, the relation $\mathcal{D}=(1 / 3) v_{E} l$ is not used explicitly. It is replaced by the Thouless relation.

A summary of our results has been given in Ref. [13]. First results for electrons were published in Ref. [15]. In the present paper we give a detailed derivation of our results, paying special attention to the necessary modifications of random matrix theory, and of the standard supersymmetry formalism. For classical waves, the latter is introduced in Ref. |14.

The random matrix model with resonances is introduced in section III. In section III, a particularly simple case is presented which paves the way for the full results given in section [V. Section $\nabla$ contains the conclusions. We use Efetov's supersymmetric generating functional [16] in the version of ref. [17].

\section{THE MODEL}

In Ref. [15, we have given a detailed physical justification for the choice of the random matrix model used to describe diffusive scattering in the presence of resonances. The arguments presented there apply to the case of electrons. Meanwhile, we have shown that the non-linear sigma models for electrons and classical waves are identical, save for the source terms 13,14. For these reasons, we keep our presentation brief. In this section we always refer to electrons. It must be understood that all arguments apply equally to light (classical waves).

In the absence of Mie resonances, a quasi one-dimensional disordered system of length $L$ for diffusive scattering of electrons is modeled |18 as consisting of many slices of length $l_{0} \ll L$ each. Within each slice, the Hamiltonian is modeled as a member of the GOE, the random-matrix ensemble with orthogonal symmetry. Neighboring slices are coupled by Gaussian-distributed uncorrelated random matrix elements. The strength of this coupling defines the diffusion constant. The first and the last slice are coupled to the channels, i.e., the asymptotic states in the leads. Elements of the scattering matrix connecting incident and outgoing channels in both leads define the transmission through the disordered region and, thus, the conductance.

To account for the presence of Mie resonances, we retain the idea of a division of the quasi one--dimensional system into slices but modify the form of the Hamiltonian $\mathbf{H}$. Within each slice, $\mathbf{H}$ is a matrix of dimension $N+m$ where $m$ is the number of Mie scatterers within each slice. This number is determined by the concentration of scatterers, and by the linear dimensions of the slice. Thus, $\mathbf{H}$ has the form

$$
\mathbf{H}=\left(\begin{array}{cc}
H_{\mathrm{GOE}} & V \\
V^{T} & E_{1} \times I_{m}+H_{\mathrm{res}}
\end{array}\right)
$$

where $T$ denotes the transpose. Here, $H_{\mathrm{GOE}}$ is a matrix of dimension $N$ belonging to the Gaussian orthogonal ensemble (GOE) of random matrices, $E_{1} \times I_{m}$ is an $m$-dimensional 
diagonal matrix with elements $E_{1}=\hbar \omega_{1}$ corresponding to the presence of $m$ Mie scatterers with equal resonance frequencies $\omega_{1}, I_{m}$ is the $m$-dimensional unit matrix, $H_{\text {res }}$ is a random matrix of dimension $m$, also a member of the GOE which describes the coupling between resonances, and $V$ is a rectangular matrix which couples the $m$ resonances to $H_{\mathrm{GOE}}$. We assume the matrices $V, H_{\text {res }}$, and $H_{\mathrm{GOE}}$ to be statistically uncorrelated. The variance of the matrix elements of $H_{\mathrm{GOE}}$ is chosen as usual: The ensemble average of $\left(H_{\mathrm{GOE}}\right)_{\mu \nu}\left(H_{\mathrm{GOE}}\right)_{\mu^{\prime} \nu^{\prime}}$ is given by $\left(\lambda^{2} / N\right)\left(\delta_{\mu, \mu^{\prime}} \delta_{\nu, \nu^{\prime}}+\delta_{\mu, \nu^{\prime}} \delta_{\nu, \mu^{\prime}}\right)$. Here, $\lambda$ has the dimension of an energy and determines the average level spacing $d_{S}$ (the same in each slice). For $m=0$ and with $\Delta(E)=\sqrt{1-(E /(2 \lambda))^{2}}$, the dependence of $d_{S}$ on energy $E$ is given by $\pi \lambda /(N \Delta(E))$ (semicircle law). At the end of the calculation, we take the limit $N \rightarrow \infty$. The ensemble average of $\left(H_{\mathrm{res}}\right)_{\mu \nu}\left(H_{\mathrm{res}}\right)_{\mu^{\prime} \nu^{\prime}}$ is written analogously as $\left(\lambda_{1}^{2} / m\right)\left(\delta_{\mu, \mu^{\prime}} \delta_{\nu, \nu^{\prime}}+\delta_{\mu, \nu^{\prime}} \delta_{\nu, \mu^{\prime}}\right)$. The strength factor $\lambda_{1}$ is chosen in such a way that the interaction between resonances results in a lifting of the degeneracy which is of the order of the mean level spacing $d_{S}(0)$ so that $\lambda_{1} \ll \lambda$. Too strong an interaction would wash out the resonance structure altogether. Finally, and without loss of generality, the rectangular matrix $V$ can be taken to be diagonal. Indeed, $V$ can be written in the form $O_{1} V_{D} O_{2}^{T}$ where $V_{D}$ is diagonal and rectangular, and where $O_{1}$ and $O_{2}$ are orthogonal matrices of dimensions $N$ and $m$, respectively. Transforming $\mathbf{H}$ with the orthogonal matrix $O=\operatorname{diag}\left(O_{1}, O_{2}\right)$, and using the orthogonal invariance of the GOE to absorb the matrices $\mathrm{O}_{1}$ into $\mathrm{H}_{\mathrm{GOE}}$ and $\mathrm{O}_{2}$ into $\mathrm{H}_{\mathrm{res}}$, respectively, we obtain a new Hamiltonian of the form of Eq. (四), but with $V$ replaced by the diagonal rectangular matrix $V_{D}$. Since we assume all scatterers to be identical, we take all diagonal matrix elements of $V_{D}$ to be equal. We assume that the diagonal matrix elements $v$ scale with $N$ in the same way as those of the matrices $H_{\mathrm{GOE}}$ and $H_{\mathrm{res}}$, so that $v^{2}=\alpha \lambda^{2} / N$ where $\alpha$ is independent of $N$. This completes the definition of the model.

It is useful to relate $\lambda_{1}$ and $v$ microscopically to the properties of the Mie resonance, and to the concentration $\mu$ of scatterers. By construction, the total coupling strength of each of the $m$ resonant states is on average given by $v^{2}+\lambda_{1}^{2}$. This quantity must, therefore, be proportional to the square of the matrix element for electromagnetic decay of the Mie resonance. Moreover, $\lambda_{1}$ must grow monotonically with increasing concentration of scatterers because an ever greater fraction of the light emitted from one resonance will be absorbed by another one, rather than scattered randomly. More precisely, consider a granule with a Mie resonance emitting a spherical wave. This wave hits an (arbitrary) second granule at distance $s$. For geometrical reasons, the cross section for absorption by excitation of the Mie resonance in this second granule is proportional to $s^{-2}$. With changing concentration, the distance $s$ between any pair of granules changes as $s^{3} \sim m^{-1}$. Hence, the coupling strength between any pair of granules (which we identify with $\lambda_{1}^{2} / m$ ) is proportional to $m^{2 / 3}$. This yields $\lambda_{1} \sim m^{5 / 6}$. This consideration suggests that the coupling between granules should be dependent upon their distance, a fact not taken into account in Eq. (1) where all resonances are coupled in the same way. Thus, using a random band matrix instead of the GOE matrix $H_{\text {res }}$ may yield a better simulation of the actual situation. We shall find, however, that the entire effect of $H_{\text {res }}$ consists in widening the peak in the mean density of states. Therefore, it does not seem worthwhile to go through the trouble of dealing with a more complicated ensemble. On the other hand, this consideration shows why it is justified not to take into account the interaction of granules located in different slices. This is the approximation we will use.

\section{ONE SLICE WITH COUPLED RESONANCES}

For pedagogical reasons we focus attention in this section to the simplest case of a single slice described by the Hamiltonian $\mathbf{H}$ given by Eq. (四). In Ref. [15], we have studied the case of a single slice with uncoupled resonances $\left(H_{\text {res }}=0\right)$. Here, we generalize this study to the case of coupled resonances $\left(H_{\text {res }} \neq 0\right)$. We calculate the mean level density and the level-level correlation function, using the supersymmetry technique of refs. [16,17. 
The advanced and retarded Green functions can be written as integrals over supervectors

$$
G_{\mu \nu}^{ \pm}(E \pm \omega)=\mp i \int \mathrm{d}[\Psi] S_{\mu}^{1} S_{\nu}^{1} \exp [ \pm i \mathcal{L}(\Psi)]
$$

where the Lagrangean is given by

$$
\mathcal{L}=\frac{1}{2} \Psi^{\dagger}(E \pm \omega \pm i \eta-\mathbf{H}) \Psi
$$

The supervectors $\Psi$ are defined by

$$
\Psi^{\dagger}=\left(S^{1}, S^{2}, \chi,-\chi^{*}\right)
$$

The vectors $S^{1}$ and $S^{2}$ have $N+m$ ordinary real components, whereas the vectors $\chi$ have $N+m$ anticommuting components. We define a source term $J=\operatorname{diag}(j, j,-j,-j)$ in graded space, where $\mathrm{j}$ is a matrix of dimension $(N+m)$ with entries $J_{\nu \mu}$, and introduce the generating functional

$$
Z^{ \pm}(E \pm \omega, J)=\int \mathrm{d}[\Psi] \exp \left[ \pm i\left(\mathcal{L}-\frac{1}{2} \Psi^{\dagger} J \Psi\right)\right] .
$$

The Green functions are obtained as functional derivatives of $Z$ with respect to $J$ at $J=0$,

$$
G_{\mu \nu}^{ \pm}=\frac{1}{4} \frac{\delta Z^{ \pm}}{\delta J_{\nu \mu}}
$$

We use this expression to calculate the average of the product of a retarded and an advanced Green function taken at different energies, $\left\langle G^{+} G^{-}\right\rangle$. This quantity (the "two-point function") plays an important role in describing average properties of random systems, such as the level-level correlation function. Except for the dimensions of the supervectors $\Psi$ and of the $\sigma$-and $Q$-matrices appearing below, and except for the occurrence of additional frequency variables, the $2 k$-point function for any positive integer $k$ is governed by an effective Lagrangean of the same type.

The generating functional $Z$ for the two-point function is given by

$$
Z(E, \omega, J)=\int \mathrm{d}[\Psi] \exp \left[\frac{1}{2} i \Psi^{\dagger} L^{1 / 2}(E+\omega L+i \eta L-\mathbf{H}-J) L^{1 / 2} \Psi\right] .
$$

We have introduced two $8(N+m)$-dimensional graded matrices, namely $L=\operatorname{diag}(I, I,-I,-I, I, I,-I,-I)$ and $J=\operatorname{diag}(j, j,-j,-j, j, j,-j,-j)$. Here, $I$ denotes the $N+m$-dimensional unit matrix. The quantity $\Psi$ now denotes a supervector with $8(N+m)$ components. All quantities are given in "advanced-retarded" notation (see Ref. [17]). We average over the two uncorrelated random ensembles $H_{\mathrm{GOE}}$ and $H_{\text {res }}$. The presence of these two ensembles causes the Hubbard-Stratonovich transformation to involve two supermatrices $\sigma$ and $\sigma_{1}$. For the generating functional, we find

$$
Z(E, \omega, J)=\int \mathrm{d}[\sigma] \exp \left[-\frac{N}{4} \operatorname{trg} \sigma^{2}-\frac{m}{4} \operatorname{trg} \sigma_{1}^{2}-\frac{1}{2} \operatorname{Trg} \log (M-\omega L-J)\right],
$$

where we define

$$
M=\left(\begin{array}{ccc}
E-\lambda \sigma & 0 & -V_{D} \\
0 & E-\lambda \sigma & 0 \\
-V_{D} & 0 & E-E_{1}-\lambda_{1} \sigma_{1}
\end{array}\right)\left\{\begin{array}{l}
m \\
N-m \\
m
\end{array}\right.
$$


Here, trg denotes the trace over an $8 \times 8$ matrix in graded space, and Trg the trace over an $8(N+m) \times 8(N+m)$ matrix in both graded and Hilbert space. The symbol d $[\sigma]$ stands for the differentials of all the independent variables in both $\sigma$ and $\sigma_{1}$. The dimensions of the block matrices in Hilbert space are indicated in Eq. (9). After taking the limit $N \rightarrow \infty$ and keeping $m$ fixed, variation with respect to $\sigma$ yields the standard saddle-point equation with a constant diagonal solution

$$
\sigma_{D}=\frac{E}{2 \lambda} \pm i \Delta
$$

where $\Delta=\sqrt{1-(E / 2 \lambda)^{2}}$. Variation with respect to $\sigma_{1}$ produces a second saddle-point equation

$$
\sigma_{1}=\frac{\lambda_{1}}{E-E_{1}-\lambda_{1} \sigma_{1}-\Gamma \sigma}
$$

where $\Gamma=v^{2} / \lambda$. Eq. (11) defines the diagonal elements of $\sigma_{1}$ in terms of those of $\sigma$. The saddle-point manifold is generated by the usual choice of signs in Eq. (10), and by transforming the resulting diagonal matrices $\sigma_{D}$ and $\sigma_{1 D}$ both by the same matrix $T$. The latter is defined in Eq. (5.28) of Ref. [17]. The resulting matrices are denoted by $Q$ and $Q_{1}$, respectively.

We integrate over the massive modes and expand the logarithm up to terms linear in $\omega$ and $J$, using the identity

$$
\exp \left[\operatorname{trg} \log \left(\begin{array}{cc}
A & B \\
B^{T} & D
\end{array}\right)\right]=\exp \left[\operatorname{trg} \log \left(\begin{array}{cc}
A-B D^{-1} B^{T} & 0 \\
0 & D
\end{array}\right)\right]
$$

and the fact that traces over terms not containing either $L$ or $J$ vanish. Introducing the diagonal graded matrix $\Lambda=\operatorname{diag}(1,1,1,1,-1,-1,-1,-1)$, we find

$$
Z(E, \omega, J)=\int \mathrm{d} \mu(t) \exp \left[-\frac{N}{\lambda} \operatorname{trg}\left[\left(Q+\frac{m \lambda}{N \lambda_{1}} Q_{1}\right)(\omega \Lambda+J)\right]\right] .
$$

The symbol $\mathrm{d} \mu(t)$ denotes the invariant measure, see Ref. [17.

The expression in Eq. (13) is formally equivalent to the standard one, obtained in the absence of resonances. This is seen by introducing a new diagonal supermatrix

$$
\sigma_{D}^{*}=\sigma_{D}+\frac{m \lambda}{N \lambda_{1}} \sigma_{1 D}
$$

and by defining a new $Q-$ matrix $Q^{*}$ by

$$
Q^{*}=T \sigma^{*} T^{-1}
$$

This leads to

$$
Z(E, \omega, J)=\int \mathrm{d} \mu(t) \exp \left[-\frac{N}{\lambda} \operatorname{trg}\left[\left(Q^{*}(\omega \Lambda+J)\right]\right]\right.
$$

Because of the presence of the $m$ resonances, the average density of states is not given directly in terms of the saddle-point solution $\sigma_{D}$ but is obtained by differentiation of the generating functional. This yields

$$
\bar{\rho}=\frac{N}{\lambda \pi} \operatorname{Im} \sigma_{D}^{*}=\frac{N \Delta}{\lambda \pi}+\frac{m}{\lambda_{1} \pi} \operatorname{Im} \sigma_{1} .
$$


The integral over $\rho$ must be equal to the dimension of the Hamiltonian, $\int \rho(E) d E=N+m$. This can be verified by a straightforward calculation. To obtain a better understanding of the form of the density of states given by Eq. (17), we consider the two limits of very small and very large $\lambda_{1}$. In the limit $\lambda_{1} \rightarrow \infty\left(\lambda_{1} \gg \Gamma\right)$, the solution of Eq. (11) to zeroth order in $\Gamma$ is given by

$$
\sigma_{1 D}=\frac{E-E_{1}}{2 \lambda_{1}}+i \Delta^{\prime}
$$

where $\Delta^{\prime}=\sqrt{1-\left(\left(E-E_{1}\right) / 2 \lambda_{1}\right)^{2}}$. For sufficiently large $\lambda_{1}$, i.e. sufficiently strong coupling between the resonances, the coupling of the Hamiltonians $H_{\mathrm{GOE}}$ and $H_{\mathrm{res}}$ can be neglected, and the density of states reduces to the sum of two semicircles

$$
\bar{\rho}=\frac{N \Delta}{\lambda \pi}+\frac{m \Delta^{\prime}}{\lambda_{1} \pi} .
$$

We see that the resonant structure is completely washed out. This was to be expected. In the opposite limit $\lambda_{1} \rightarrow 0$ (uncoupled resonances), it is easily seen that we retrieve our earlier result for uncoupled resonances [15]

$$
\overline{\rho(E)}=\frac{\Delta(E) N}{\lambda \pi}+m \frac{\Gamma \Delta / \pi}{\left(E-E_{1}-(E / 2 \lambda) \Gamma\right)^{2}+(\Gamma \Delta)^{2}},
$$

where $\pi \lambda /(N \Delta)$ is the mean level spacing $d_{S}$ in the absence of resonances. The second term in Eq. (20) has area $m$, peak height $m /(\pi \Gamma \Delta)$, and Lorentzian shape. As expected, Eq. (20) shows a resonance enhancement of the mean level density, centered at the energy $E_{1}$ of the $m$ resonances. The total width $(\Gamma \Delta)$ is determined by the strength $v$ of the coupling to the random scatterers. We recall the relation $v^{2}=\alpha \lambda^{2} / N$ and find $\Gamma \Delta=\alpha \lambda \Delta / N=(\alpha / \pi) d_{S}$. This shows that $\Gamma$ is of the order of the mean level spacing $d_{S}$. For light scattering, we have $\alpha \gg 1$. For formal reasons, we also consider the case $\alpha \sim 1$ in this paper.

The expression for the width $w$ of the Lorentzian in Eq. (20) can be derived from a different point of view with the help of the golden rule. With $\mid r>$ a resonance state and $\mid i>$ a state in the $N$-dimensional subspace of Hilbert space comprising the GOE part of $\mathbf{H}$, we find, averaging over the states labelled $\mid i>$,

$$
w=\pi \frac{1}{N} \sum_{i}^{N}<r|V| i>^{2} \delta\left(E_{i}-E_{1}\right) .
$$

Since $\sum_{i}^{N} \delta\left(E_{i}-E_{1}\right)=N \Delta / \pi \lambda$, we immediately find $w=\Gamma \Delta$, i.e. the result we obtain using the supersymmetry formalism.

Using the explicit form of the matrix $Q^{*}$ in Eq. (15), we can write the effective action in the exponent of Eq. (16) in the form

$$
-i \pi \frac{\omega}{d_{\mathrm{eff}}} \operatorname{trg} T^{-1} \Lambda T \Lambda
$$

where $d_{\text {eff }}(E)$ is the inverse of the average level density $\overline{\rho(E)}$ given in Eq. (17). We note that, aside from the definition of $d_{\text {eff }}$, the form of this expression is the same as in the absence of any resonances. Taking into account the fact that we can justify the second saddle-point equation only for $\omega \ll \Gamma$, we conclude that (as in the case of uncoupled resonances [15]) the only change in the level-level correlation function caused by the presence of resonances is due to the rescaling of the mean level density. Fig. 1 shows the result for a set of parameters given in the figure caption. 


\section{CONDUCTANCE OF A QUASI ONE-DIMENSIONAL SAMPLE}

In this Section we consider a quasi one-dimensional system coupled to two perfect leads. As mentioned in Sec. II, we consider this system as consisting of $K$ slices labelled $i$ or $j$ with $i, j=1, \ldots, K$. Within each slice, the Hamiltonian has the form given in Eq. (2). Neighboring slices $(|j-i|=1)$ are coupled by random matrices $H_{\mu \nu}^{i j}$ with Gaussiandistributed uncorrelated matrix elements with zero mean value and a second moment given by $\left(p^{2} / N\right)\left(\delta_{\mu, \mu^{\prime}} \delta_{\nu, \nu^{\prime}}+\delta_{\mu, \nu^{\prime}} \delta_{\nu, \mu^{\prime}}\right)$. For reasons given earlier, we assume that only the random parts of the Hamiltonians in neighboring slices are coupled.

In the case of a single slice, we have seen that the mutual coupling of resonances leads only to a quicker broadening of the resonances than would be the case otherwise, and finally to the disappearance of the resonance enhancement effect. With the intention of simplifying the presentation we, therefore, now consider the situation where the resonances are not coupled even within one slice $\left(\lambda_{1}=0\right)$.

The coupling between the left (right) lead and the first (last) slice $(j=1$ or $K$ ) is described by the real matrix elements $W_{a \mu}^{j}$ [18]. Here, the index $a$ labels the channels in the leads. We assume that the dependence on energy of these matrix elements is negligible. We are interested in the influence of the resonances on the coupling to the channels. Therefore, we allow for a direct coupling between leads and resonances. Hence, the index $\mu$ runs from 1 to $N+m$. Following Ref. [18], we assume for $j=1, K$ the orthogonality relations

$$
\sum_{\mu=1}^{N+m} W_{a \mu}^{j} W_{b \mu}^{j}=(N+m)\left(v_{a}^{j}\right)^{2} \delta_{a b} .
$$

We use the many-channel Landauer formula

$$
g=\sum_{a, b}\left(\left|S_{a b}^{L R}\right|^{2}+\left|S_{a b}^{R L}\right|^{2}\right)
$$

which expresses the conductance $g$ at zero temperature in terms of the elements $S_{a b}^{L R}$ of the scattering matrix associated with the total Hamiltonian. Here $R$ and $L$ stand for the right and left leads, respectively. The scattering matrix has the form

$$
S_{a b}^{c d}=\delta^{c d} \delta_{a b}-2 i \pi \sum_{\mu, \nu} W_{a \mu}^{c}\left[D^{-1}\right]_{\mu \nu}^{c d} W_{b \nu}^{d}
$$

where the inverse propagator $D$ is a matrix of dimension $K \times(N+m)$ given by

$$
D_{\mu \nu}^{i j}=E \delta_{\mu \nu} \delta^{i j}-H_{\mu \nu}^{i j}+i \pi \sum_{a, c} W_{a \mu}^{c} W_{a \nu}^{c}\left(\delta_{i 1} \delta_{c L}+\delta_{i K} \delta_{c R}\right) \delta^{i j}
$$

where $i, j=1,2, \ldots, K$ and $\mu, \nu=1,2, \ldots, N+m$. The indices $(c, d)$ on $D^{-1}$ in Eq. (25) stand for $(1,1)$ if $(c, d)=(L, L)$, for $(1, K)$ if $(c, d)=(L, R)$, etc. The energy $E$ equals the Fermi energy and eventually will be taken to have the value zero.

To calculate the ensemble average of $g$, we follow Refs. 177 and 18 and express the conductance in terms of a supersymmetric generating functional. We only indicate the steps where we deviate from the references just given. We define a generating functional $Z$ for the conductance $g$, in a way similar to that presented in the previous section.

$$
Z(J)=\int \mathcal{D}[\Psi] \exp \left[\frac{1}{2} i \Psi^{\dagger} L^{1 / 2}(E+i Q(j)+i \eta L-\mathbf{H}+\mathbf{J}(j)) L^{1 / 2} \Psi\right] .
$$

The definitions of the source term $\mathbf{J}$ and of the matrix $Q$ may be found in Ref. [18]. Suffice it to say that both $\mathbf{J}$ and $Q$ are different from zero only for the first and the last slice (only 
these two slices are coupled to the leads), and that $Q$ essentially comprises the last term on the rhs of Eq. (26). After averaging and the Hubbard-Stratonovich transformation, the generating functional for the conductance $g$ has the form

$$
\overline{Z(J)}=\int \mathrm{d}[\sigma] \exp \left[-\frac{1}{4} \sum_{i, j} g_{i j} \operatorname{trg} \sigma(i) \sigma(j)-\frac{1}{2} \sum_{j} \operatorname{Trg} \log (M(j)+i Q(j)+\mathbf{J}(j))\right] .
$$

As before, the indices $i, j$ label the slices. The integration extends over $K$ independent $\sigma$-matrices. A matrix $g=P^{-1}$ of dimension $K$ (not to be confused with the conductance) is introduced, where $P_{i j}=(1 / N)\left[\delta_{i j}+\left(p^{2} / \lambda^{2}\right)\left(\delta_{i, j+1}+\delta_{i+1, j}\right)\right]$. For each $j$, the matrix $M(j)$ has the form given in Eq. (9) with $\lambda_{1}=0$.

Using the saddle-point approximation (valid for $N \gg 1$ ), we determine the mean level density and show that the resonant structure found for a single slice remains qualitatively unchanged. Omitting the source term, and taking the limit $m / N \rightarrow 0$, we obtain the saddle-point equation

$$
\sum_{i} g_{i j} \sigma(i)=\lambda N \frac{1}{E-\lambda \sigma(j)}
$$

We note that this equation is identical to the one which would obtain in the absence of all resonances. It was shown in Ref. [18] that the unique diagonal solution of Eq. (29) has the form

$$
\sigma_{D}(j)=r^{j} I_{4}-i \Delta^{j} L
$$

Assuming weak coupling between the slices, we find for the total density of states in the entire sample in zeroth order in $p^{2} / \lambda^{2}$

$$
\bar{\rho}^{\mathrm{tot}}=\sum_{j=1}^{K} \bar{\rho}(j)
$$

where $\bar{\rho}(j)$ is given by Eq. (20) with $r^{j}$ substituted for $E / 2 \lambda, \Delta^{j}$ for $\Delta$. This result shows that the Lorentzian part in the average level density which is due to the contribution from the resonances, is preserved. The importance of this statement lies in the fact that the conclusions valid for a single slice apply likewise to the entire sample.

To see this, we calculate the two-point function. We follow Ref. [18 and parameterize $\sigma(j)$ as $\sigma(j)=\left(T^{j}\right)^{-1} \sigma_{D}(j) T^{j}$ with

$$
T^{j}=\left(\begin{array}{cc}
\sqrt{1+t_{12}^{j} t_{21}^{j}} & i t_{12}^{j} \\
-i t_{21}^{j} & \sqrt{1+t_{21}^{j} t_{12}^{j}}
\end{array}\right)
$$

We emphasize that in spite of the presence of the $m$ resonances in each slice, the form of the matrices $\sigma(j)$ is the same as in Ref. [18 (absence of resonances). We recall that the matrices $Q$ and $\mathbf{J}$ differ from zero for the first and the last slice only. From the identity Eq. (12) and the fact that $\operatorname{Trg} \log \left(E-E_{1}\right)=0$, we conclude that the logarithmic term in the exponent of Eq. (28) vanishes unless $j=1$ or $j=K$. Except for these two terms, the resulting form of the generating functional is identical to the one given in Ref. [18]. Moreover, the terms with $j=1$ or $K$ differ from zero only because of the presence of the matrices $Q$ and $\mathbf{J}$ both of which involve the coupling to the channels. These results show that the presence of resonances does not affect the behavior of the average conductance in the bulk, but only the way it is coupled to the leads. It was shown in Ref. [18] that for 
sufficiently long samples (where the Thouless energy is smaller than the decay width defined by the coupling to the leads), the influence of the coupling to the leads is immaterial for the behavior of the average conductance. In practice, this condition is met for samples with lengths exceeding a few elastic mean free paths. Using these facts, we conclude that for typical samples, the average conductance is the same with and without resonances. Since the average conductance is calculated at fixed frequency, this conclusion holds likewise for electrons and for light (provided the frequency in the latter case is sufficiently high). This is also true for the calculation of the transmission coefficients given below.

Before addressing the influence of the resonances on the source term and on the coupling to the channels, we turn attention briefly to the intensity autocorrelation function $\overline{g(E) g(E+\omega)}$. We do not describe the calculation of this quantity (a four-point function) in any detail. It is not difficult to see, however, that the effective action for this quantity also has the form given in Eq. (28), except for the following changes. (i) The dimensions of the matrices $\sigma(j)$ are doubled. (ii) For each value of $j=1, \ldots, K$, an extra term $-\omega \Lambda$ appears under the logarithm. Here, $\Lambda$ is the sixteen-dimensional analogue of the matrix defined above Eq. (13).

What are the changes caused by the appearance of this extra term under the logarithm? We neglect the source term and the $Q$ matrix. For each value of $j$, we expand the logarithm in powers of $\omega$, keeping only the linear term. (The term of zeroth order vanishes). After some simple algebra, we find that the result has a form analogous to expression (22),

$$
-i \pi \frac{\omega}{d_{\mathrm{eff}}} \sum_{j=1}^{K} \operatorname{trg}\left(T^{j}\right)^{-1} \Lambda T^{j} \Lambda .
$$

It has to be borne in mind that the $T$ 's are now matrices of dimension sixteen. Here, $d_{\text {eff }}$ is defined as the inverse of the mean level density $\bar{\rho}$ per slice, see Eq. (20), but with $r^{j}$ substituted for $E / 2 \lambda, \Delta^{j}$ for $\Delta$, see Eq. (30). We assume that $\bar{\rho}$ has the same value in each slice. Comparing our result with what would apply in the absence of resonances, we see that in each slice, $1 / d$ is replaced by $1 / d_{\text {eff }}$. For Efetov's effective action (obtained in the continuum limit where the number of slices tends to infinity while their individual length shrinks to zero) this means that the frequency $\omega$ is multiplied by the total level density $\bar{\rho}^{\text {tot }}$ defined in Eq. (31). This is the claim made in Ref. [13] and in the Introduction to the present paper. In contradistinction to the average conductance, the four-point correlation function is influenced by the presence of resonances: The frequency $\omega$ occurs only in the combination $\omega \bar{\rho}^{\text {tot }}$. The result for $\mathcal{D}$ is shown in Figure 1 of Ref. [13]. The argument can easily be extended to correlation functions of higher order.

The conclusions drawn in the last three paragraphs contain the main results of this work. It remains to calculate the influence of the resonances on the coupling to the leads. For reasons given above, this may be considered a somewhat academic exercise. However, experiments on short samples would be able to test the predictions made in this way.

The terms describing the coupling to the channels are part of the matrices $Q$ and $\mathbf{J}$ under the logarithm in Eq. (28). We recall that both these terms are different from zero only for the first $(j=1)$ and last $(j=K)$ slices. In expanding the logarithm, we have to keep terms of any order in $i Q(j)$ but only of zeroth and first order in $\mathbf{J}$. Here, we focus attention on the terms of zeroth order in $\mathbf{J}$. (The calculation of the first-order terms proceeds analogously). After calculating the trace over $\mu$ in each term of the series by using the orthogonality relation Eq. (23) for $j=1(K)$ and resumming the result, we use the saddle-point solution for the first and the last slices, the parameterization of $\sigma$ given in Eq. (32), and introduce the quantities $\Gamma_{a}=V_{a}^{2} / \lambda$. After some algebra, we find for $j=1, K$

$$
-\frac{1}{2} \sum_{a} \operatorname{Trg} \log \left(1+\frac{1}{2} T_{a} t_{12}^{j} t_{21}^{j}\right)
$$

where $T_{a}$ is the "sticking probability" in channel $a$, given by 


$$
T_{a}=\frac{4 \Delta\left(-\frac{\Gamma_{a}^{2}}{\lambda} \frac{\Gamma-\frac{E}{\lambda}\left(E-E_{1}\right)}{\left(E-E_{1}-\frac{E}{2 \lambda} \Gamma\right)^{2}+\Gamma^{2} \Delta^{2}}+\frac{W_{a}}{\lambda}\right)}{\left(1+\frac{W_{a}}{\lambda} \Delta-\frac{\Gamma_{a}^{2}}{\lambda} \Delta \frac{\Gamma-\frac{E}{\lambda}\left(E-E_{1}\right)}{\left(E-E_{1}-\frac{E}{2 \lambda} \Gamma\right)^{2}+\Gamma^{2} \Delta^{2}}\right)^{2}+\left(\frac{E}{2 \lambda} \frac{W_{a}}{\lambda}-\frac{\Gamma_{a}^{2}}{\lambda} \frac{\left(E-E_{1}\right)\left(1-\frac{1}{2}\left(\frac{E}{\lambda}\right)^{2}+\frac{E}{2 \lambda} \Gamma\right.}{\left(E-E_{1}-\frac{E}{2 \lambda} \Gamma\right)^{2}+\Gamma^{2} \Delta^{2}}\right)^{2}} .
$$

The sticking probabilities $T_{a}$ are related to the matrix elements $\overline{S_{a a}^{c c}}$ of the average scattering matrix by $T_{a}=1-\left|\overline{S_{a a}^{c c}}\right|^{2}$. This means that $T_{a}$ measures that part of the flux which is not re-emitted instantaneously into the same channel but which experiences multiple scattering in the disordered sample. Eq. (35) very clearly shows that the sticking probabilities are affected by a direct coupling between resonances and channels.

\section{CONCLUSIONS}

It was shown in Ref. [18 that the conductance of a short sample is proportional to the product of two terms, each term being given by the sum of the sticking probabilities of the channels in one of the two leads. Thus, the conductance of a short sample is determined by the coupling to the leads, whereas the conductance of a long sample is entirely determined by the matrix $g_{i j}$ appearing in Eq. (28). This implies that as long as there is no direct coupling between resonances and leads, the conductances of both short and long samples are unchanged. When the resonances are coupled to the channels, however, the conductance of a short sample can be changed drastically at frequencies near the resonance frequency, whereas the corresponding change of the conductance of a long sample is expected to be small. It would be interesting to search for this effect experimentally.

On the other hand, the intensity autocorrelation function is always modified by the presence of resonances, both for short and for long samples. This fact follows directly from our calculation of the diffusion constant $\mathcal{D}$ (which shows a resonance dip) but is most easily seen by using an argument based on the Thouless relation. This argument was given in the Introduction and is not repeated here. The argument is fully corroborated by our analytical results.

It was emphasized above that the effective Lagrangean for electrons and for scalar waves (light) is the same, both in the absence and in the presence of Mie resonances, provided that the usual condition $k l \gg 1$ is met. Here, $k$ is the wave number and $l$ the elastic mean free path. In view of remarks in the literature that the strong effect of Mie resonances upon light scattering is absent in the case of electrons, we wish to clarify this statement. In the case of scalar waves (light), there are two different and unrelated effects which contribute towards the energy dependence of the potential. The first of these is due to the dependence on $k$ of the term $k^{2} \epsilon(\vec{r})$ in the scalar wave equation. Here, $\epsilon$ is the space-dependent dielectric constant. The disorder potential $V$ in the Schrödinger equation has no analogous dependence on wave number or energy. However, the change with $k$ of $k^{2} \epsilon(\vec{r})$ is small on the scale of the mean level spacing. This is why the non-linear sigma models for electrons and for scalar waves are identical, even though the Ward identities for the two wave equations differ. Second, the presence of Mie resonances causes the drastic modification of the intensity autocorrelation function for light scattering discussed above. The equality of the nonlinear sigma models for scalar and for Schrödinger waves implies that a correspondingly large modification of the intensity autocorrelation function would also occur for electrons if it were possible to design a system in which electrons are scattered both diffusively and via Mie-like resonances. To the best of our knowledge, such a system does not exist at present, and the stated analogy makes a prediction which cannot be checked experimentally.

To compare with experiment, we note: (i) For classical waves in a uniform medium in $d$ dimensions, the density of states is proportional to $\epsilon^{d / 2}$ where $\epsilon$ is the dielectric constant of the medium. Increasing the number of resonating spheres, we increase the effective dielectric constant of the medium. Hence, $\lambda$ should decrease with increasing $\mu$. (ii) As shown in Sec. III, $\lambda_{1}$ is expected to increase monotonically with $\mu$. Both these points affect the dependence on concentration of $d_{\text {eff }}$ and, therefore, of $\mathcal{D} \sim d_{\text {eff }}$. With increasing $\mu$, point (i) causes a 
decrease of $\mathcal{D}$ far away from the resonance dip, and point (ii) a widening the resonance dip. Fig. 1 of Ref. [13] shows $\mathcal{D}$ versus energy for different concentrations. In agreement with the experimental results [3, 12], we find a deep dip in the diffusion constant at the resonance energy. It is caused by the second term in Eq. (20) and is particularly pronounced at low concentrations of the resonant scatterers. As the concentration increases, the dip is smeared out. At the same time, the value of $\mathcal{D}$ outside the resonance dip decreases.

\section{ACKNOWLEDGMENTS}

Discussions with Dr. A. Müller-Groeling led to the central idea of how to treat the resonances in the framework of random matrix theory. V. K. appreciates very useful discussions with Dr. Y. Fyodorov and gratefully acknowledges the support of a MINERVA Fellowship. B. E. wishes to thank Prof. A. Nourreddine for valuable discussions. 


\section{REFERENCES}

* Also at Université Chouaïb Doukkali, Faculté des Sciences, El Jadida, Morocco.

[1] Y. Imry, Introduction to Mesoscopic Physics, (New York, Oxford, Oxford University Press, 1997).

[2] For a review, see Scattering and Localization of Classical Waves in Random Media, edited by Ping Sheng (World Scientific, Singapore, 1990); Photonic Band Gaps and Localization, edited by C. M. Sokoulis (Plenum, New York, 1993).

[3] M. van Albada, B. A. van Tiggelen, A. Lagendijk, and A. Tip, Phys. Rev. Lett. 66 (1991) 3132; B. A. van Tiggelen, A. Bart, A. Lagendijk and A. Tip, Phys. Rev. B 45 12233 (1992).

[4] E. Kogan and M. Kaveh, Phys. Rev. B 4610636 (1992).

[5] G. Cwilich and Y. Fu, Phys. Rev. B 4612015 (1992).

[6] J. Kroha, C. M. Soukoulis and P. Wölfle, Phys. Rev. B 4711093 (1993).

[7] Yu. N. Barababenkov and V. Ozrin, Phys. Rev. Lett. 691364 (1992).

[8] B. A. van Tiggelen, A. Lagendijk and A. Tip, Phys. Rev. Lett. 711284 (1993).

[9] B. A. van Tiggelen and A. Lagendijk, Europhys. Lett. 23311 (1993).

[10] K. Busch and C. M. Soukoulis, Phys. Rev. Lett. 75 (1995) 3442.

[11] K. Busch and C. M. Soukoulis, Phys. Rev. B 54 (1996) 893.

[12] N. Garcia, A. Z. Genack and A. A. Lisyansky, Phys. Rev. B 46, 14475 (1992); A. A. lisyansky et al., in Photonic Band Gaps and Localization, edited by C. M. Soukoulis (Plenum, New York, 1993) p. 171.

[13] B. Elattari, V. Kagalovsky, and H.A. Weidenmüller, submitted to Europhys. Lett. (1997).

[14] B. Elattari, V. Kagalovsky, and H.A. Weidenmüller, submitted to Phys. Rev. E. (1997).

[15] B. Elattari, V. Kagalovsky, and H.A. Weidenmüller, Nucl. Phys. A 60686 (1996).

[16] K. B. Efetov, Adv. Phys. 32 (1983) 53.

[17] J. J. M. Verbaarschot, M. R. Zirnbauer, and H. A. Weidenmüller, Phys. Rep. 129 (1985) 387.

[18] S. Iida, H. A. Weidenmüller, and J. Zuk, Phys. Rev. Lett. 64 (1990) 583 and Ann. Phys. (N.Y.) 200 (1990) 219. 


\section{FIGURES}

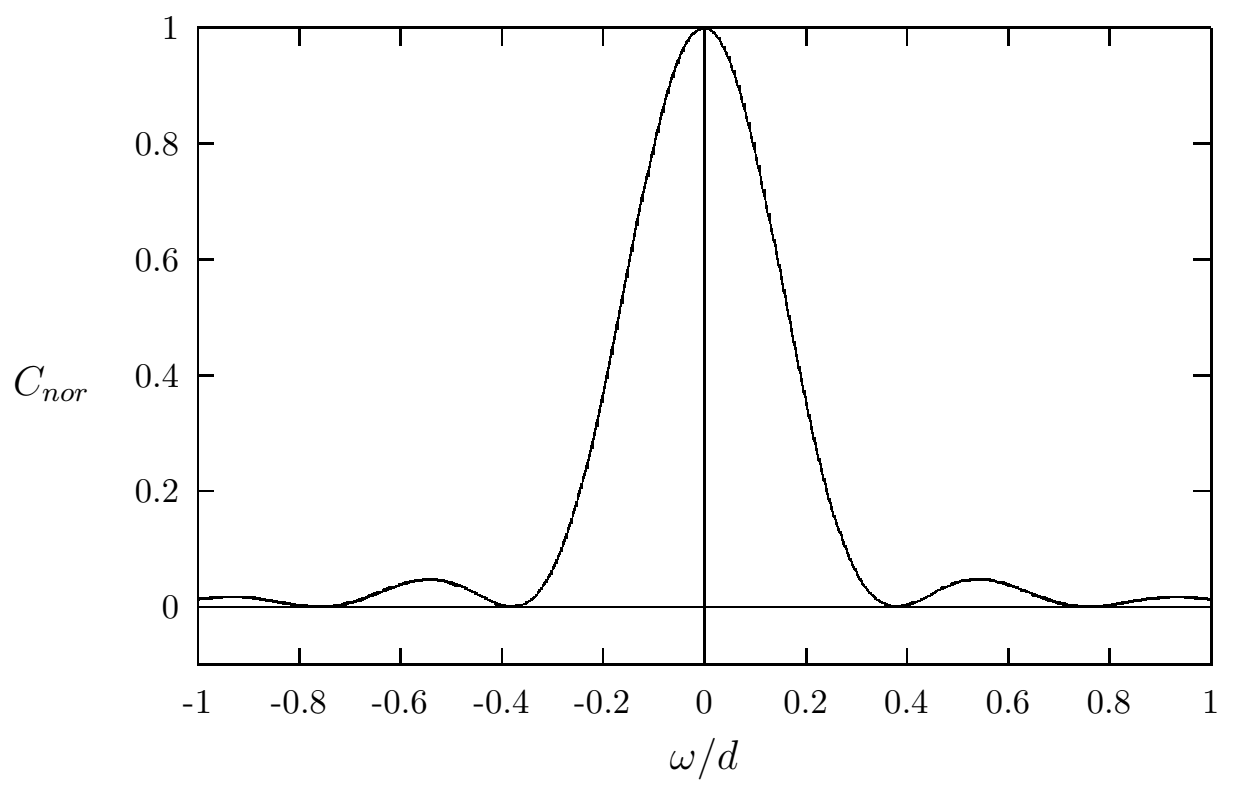

FIG. 1. The exact normalized level-level correlation function for $m=5, \Gamma=5 d, E=E_{1}=0$ and rescaled correlation function for a pure GUE given by $\sin ^{2}\left(2 \pi \omega / d_{\text {eff }}\right) /\left(2 \pi \omega / d_{\text {eff }}\right)^{2}$. 